Doug Geisler, Eva K. Grebel, and Dante Minniti, eds.

\title{
Ultra-compact Dwarf Galaxies: a New Class of Compact Stellar System Discovered in the Fornax Cluster
}

\author{
Michael Drinkwater \\ School of Physics, University of Melbourne, Victoria 3010, Australia
}

Kenji Bekki, Warrick Couch

School of Physics, Univ. of New South Wales, Sydney 2052, Australia

Steve Phillipps

Department of Physics, University of Bristol, Bristol BS8 1TL, UK

Bryn Jones

School of Physics, University of Nottingham, Nottingham NG7 2RD, UK

Michael Gregg

Department of Physics, University of California, Davis, CA 95616, USA

\begin{abstract}
We have used the 2dF spectrograph on the Anglo-Australian Telescope to obtain a complete spectroscopic sample of all objects in the magnitude range, $16.5<b_{J}<19.8$, regardless of morphology, in an area centred on the Fornax Cluster of galaxies. Among the unresolved targets are five objects which are members of the Fornax Cluster. They are extremely compact stellar systems with scale lengths less than 40 parsecs. These ultra-compact dwarfs are unlike any known type of stellar system, being more compact and significantly less luminous than other compact dwarf galaxies, yet much brighter than any globular cluster.
\end{abstract}

\section{The Fornax Cluster Spectroscopic Survey}

Our 2dF Fornax Cluster Spectroscopic Survey (FCSS; Drinkwater et al. 2000a) was designed to make the most complete census possible of low-luminosity galaxies in the Fornax Cluster. Having shown (Drinkwater \& Gregg 1998) that compact, high surface brightness dwarf cluster galaxies were missed in previous work, we took the unusual step of observing all objects in each $2 \mathrm{dF}$ field, both resolved ("galaxies") and unresolved ("stars"). In this way we avoided any morphological bias as to what a cluster galaxy should look like. Observing the "stars" in each field greatly increases the number of targets: over $50 \%$ of objects with $16.5<b_{J}<19.8$ are stars. However the flexibility of $2 \mathrm{dF}$ means this only led to a small increase in total observing time as we scheduled the stars at times when we could not usefully observe galaxies, such as twilight or through cloud. 


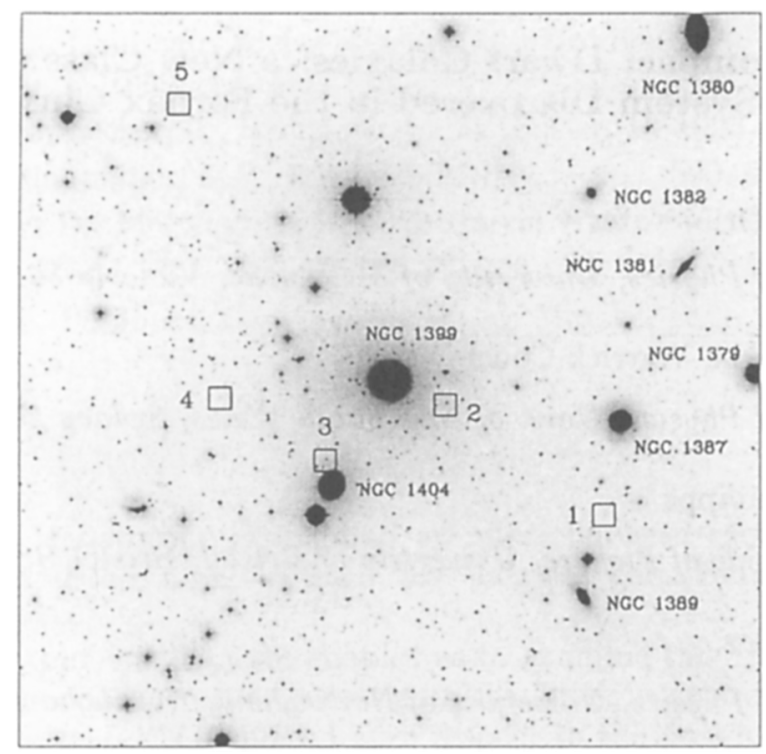

Figure 1. R-band UKST image of the central 1 degree region of the Fornax Cluster. The positions of the UCDs are shown by squares.
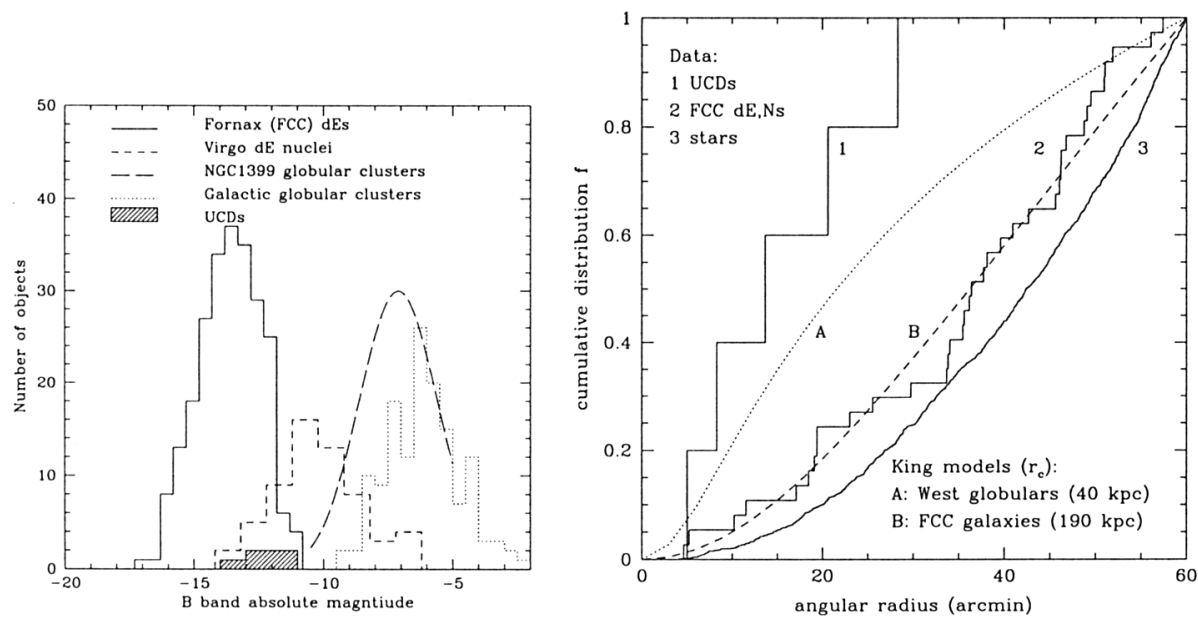

Figure 2. Left: Absolute magnitude distribution of UCDs compared to Fornax Cluster dEs (FCC, Ferguson 1989), the nuclei of Virgo Cluster dE,Ns (Binggeli \& Cameron 1991), a fit to globular clusters around NGC 1399 (Bridges et al. 1991) and Galactic globular clusters (Harris 1996). Right: Cumulative radial distribution of the new compact objects compared to the predicted distribution for intra-cluster globular clusters (West et al. 1995) and the profile fit to the distribution of all FCC members. Also shown is the distribution of all nucleated dwarfs in the FCC and the stars observed in our survey. 


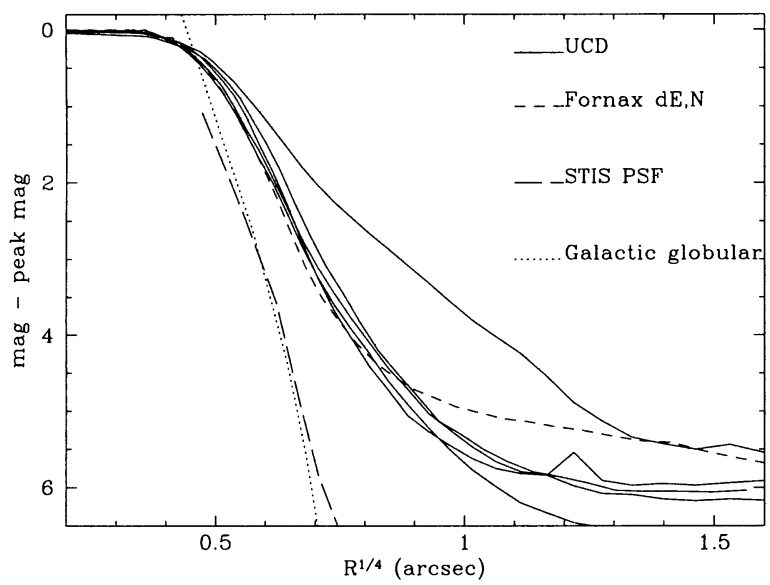

Figure 3. Radial profiles of the UCDs measured with STIS compared to the centre of a nucleated dwarf elliptical galaxy in the Fornax cluster measured with the same setup. The profiles are all normalised to their peak intensity. Also shown are the instrumental PSF from the STIS manual and the profile of a galactic globular cluster scaled to the distance of Fornax but not convolved with the PSF.

\section{Properties of Ultra-compact Dwarfs}

The highlight of our results to date has been the discovery of a new population of Fornax Cluster dwarf galaxies so compact they were previously mistaken for Galactic stars (Drinkwater et al. 2000b). The locations of these objects in the cluster is shown in Fig. 1. The 2dF discovery spectra of these compact objects show them to be old stellar systems - no Balmer lines are detected-but we await higher resolution spectra for more detailed classifications. These "ultracompact dwarf" (UCD) galaxies are unlike any known type of stellar system. They are smaller and more concentrated than any known dwarf galaxy, but are 2-3 magnitudes more luminous than the largest Galactic globular clusters. Their luminosities are compared with other stellar systems in Fig. 2(a). The only known objects they resemble both in luminosity and morphology are the nuclei of nucleated dwarf elliptical galaxies, but without any surrounding low surface brightness envelope. The radial distribution of the UCDs given in Fig. 2(b) shows that they are concentrated to the centre of the cluster, much more than the general population of cluster galaxies or even the nucleated dwarfs. The only distribution on the Figure that resembles that of the UCDs is that proposed for globular clusters formed in situ in the intra-cluster medium as suggested by West et al. (1995).

We have obtained high-resolution images of the UCDs with the STIS instrument on the Hubble Space Telescope (HST) to measure their sizes. From a preliminary analysis of our HST data we have calculated radial profiles of the UCDs shown in Fig. 3. Four of the UCDs and a dE,N nucleus we observed for comparison all have very similar profiles: they are resolved by STIS with 

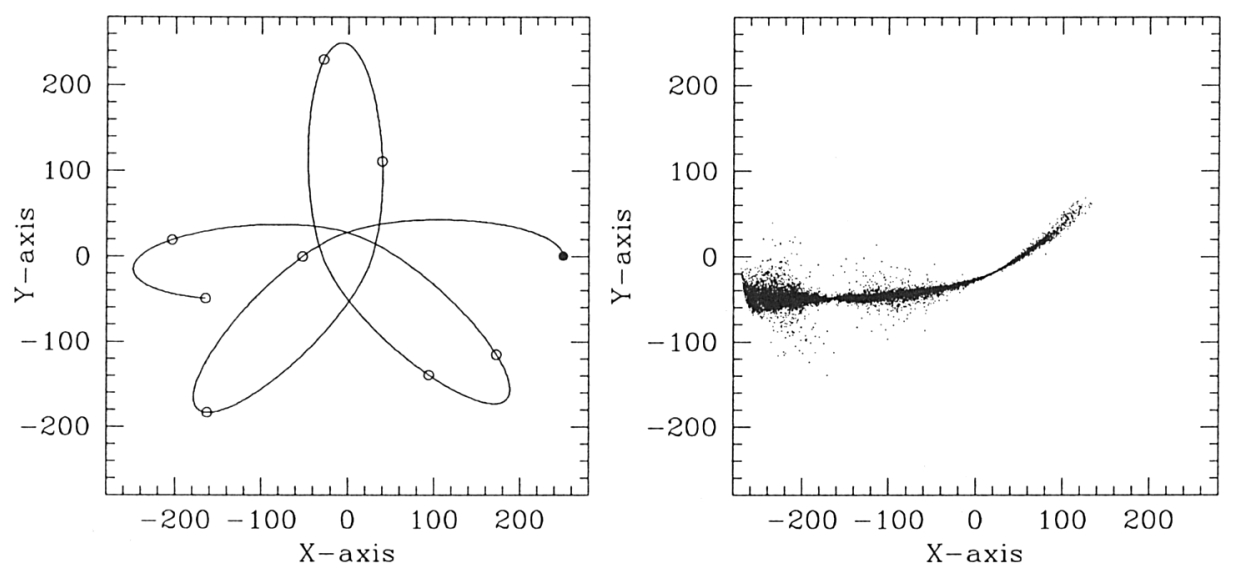

Figure 4. Numerical simulation of the formation of a UCD by tidal stripping of a nucleated dwarf elliptical in an orbit passing close to the central cluster galaxy NGC 1399 (see Bekki et al. 2001 for details). Left: The orbit of the simulated dwarf around NGC 1399 (at the origin; the scale is in units of $0.8 \mathrm{kpc}$, so the frame is $450 \mathrm{kpc}$ across). The filled circle represents the initial position of the dwarf and the orbital evolution is indicated by open circles indicating successive time intervals of $4.7 \times 10^{8} \mathrm{yr}$. Right: the final mass distribution of the dwarf galaxy at a time of $3.8 \mathrm{Gyr}$. Owing to the strong tidal field of NGC 1399, the dwarf is greatly stretched and most of the outer stellar component of the dwarf is tidally stripped away. The core of the dwarf, however, remains intact.

FWHM $=11-16 \mathrm{pc}$ and de Vaucouleurs effective radii of $R_{e} \approx 10 \mathrm{pc}$. One of the UCDs (number 3; see Fig. 1) is larger than the rest (FWHM=17 pc, $R_{e} \approx 300$ pc). The UCDs are all significantly larger than globular clusters, but they do have similar physical sizes and profiles to the dwarf elliptical nucleus we observed (except for number 3).

\section{What are Ultra-compact Dwarf galaxies?}

We are considering three hypotheses to explain the nature and origin of the UCDs. First they may simply be very massive star clusters, such as the intracluster globular clusters proposed by West et al. (1995), although their luminosities remain very high, well beyond those of galactic globular clusters. Perhaps we are seeing the very high-luminosity tail of the globular cluster population associated with NGC 1399.

Our second hypothesis is that these are the stripped nuclei of dwarf galaxies. Previous studies of the very large globular cluster population associated with central cluster galaxies like NCG 1399 in the Fornax Cluster have invoked tidal stripping of dwarf galaxies to explain the globular cluster populations. This process was simulated in some detail by Bassino et al. (1994) who predicted the formation of even larger remnants that might resemble the UCDs. We have per- 
formed numerical simulations of tidal stripping of nucleated dwarf galaxies in close orbits around the central galaxy of the cluster, NGC 1399 (Bekki, Couch \& Drinkwater 2001). The simulations show that this process is a feasible mechanism for the formation of UCDs. The result of one simulation is shown in Fig. 4. The simulations show that dwarfs in close orbits around NGC 1399 can have their outer stellar envelopes removed in a few orbits (a few Gyr), leaving just the nuclei which we have shown are of similar size to the UCDs.

Finally they may represent an entirely new type of compact galaxy. In this case they might be miniature compact ellipticals (like M32). The main test we plan of these different hypotheses is to measure the internal dynamics of the UCDs using high-resolution VLT spectra to measure their velocity dispersions. We will combine these with our HST measurements of their sizes to make estimates of their virial masses and hence their mass-to-light ratios $(\mathrm{M} / \mathrm{L})$. We would expect star clusters to have low $\mathrm{M} / \mathrm{L}$ values, whereas compact galaxies would have higher $\mathrm{M} / \mathrm{L}$ values, if we assume that they have some dark matter content-there is still no clear prediction on the existence of dark matter halos at these low mass limits.

Acknowledgments. This project has only been possible thanks to the superb performance of the $2 \mathrm{dF}$ facility, for which we wish to thank the many staff of the Anglo-Australian Observatory involved in its support. We also acknowledge the assistance of our colleagues in the FCSS team. Their names can be found on our web site at http://astro.ph.unimelb.edu.au/data/ where we also provide public access to the data from our first field.

\section{References}

Bassino, L.P., Muzzio, J.C., Rabolli, M. 1994, ApJ, 431, 634

Bekki K., Couch W.J., Drinkwater M.J., 2001, ApJ, 522, L105

Binggeli, B., Cameron, L.M., 1991, A\&A, 252, 27

Bridges, T.J., Hanes, D.A., Harris, W.E., 1991, AJ, 101, 469

Drinkwater, M.J., Gregg, M.D., 1998, MNRAS, 296, L15

Drinkwater M.J., et al. 2000a, A\&A, 355, 900

Drinkwater M.J., Jones, J.B., Gregg, M.D., Phillipps, S., 2000b, PASA, 17, 227

Ferguson H.C., 1989, AJ, 98, 367

Harris, W.E., 1996, AJ, 112, 1487

West, M.J., Cote, P., Jones, C., Forman, W., Marzke, R.O. 1995 ApJ 453 L77

\section{Discussion}

J. Forte: Your limiting magnitude, assuming $B-R \sim 1.5$, means $R \sim 18.2$ and this is the magnitude where we begin to see a unimodal distribution that seems to be the faint tail of your distribution (as mentioned by Richtler in his talk, based on photometry by Dirsch et al.). Any comment on this? 
M. Drinkwater: It will be very interesting to investigate this intermediate region around $M_{B}=-11$, so I look forward to seeing spectroscopic measurements of the objects you describe. However, I think they are very much more closely associated with NGC 1399 than the UCDs I have been finding.

M. Hilker: First, a question: What is the tiemscale for the disruption of dE,N's in the models? How fast are they dissolved? And secondly a comment: In a spectroscopic survey with the LCO $2.5 \mathrm{~m}$ telescope, we investigated the existence of compact objects at the faint limit of the known UCD sample. So far, we found only 1 candidate out of 80 objects. It would be interesting to know how many objects fill the luminosity gap between GCs and UCDs.

M. Drinkwater: The stripping is mostly completed after 4 passages close to the central galaxy - in about 4 Gyr. As for your comment, yes, I agree: let's do it!

$J$. Frogel: What's the dispersion in radial velocity compared to that of Fornax galaxies? Over the magnitude range you have found these UCDs, how complete are you?

M. Drinkwater: The velocity dispersion of the UCDs is about $250 \mathrm{~km} / \mathrm{s}$, small compared to that of the cluster galaxies $(374 \mathrm{~km} / \mathrm{s})$ but I'm not sure if the difference is significant. Our spectroscopy is $80 \%$ complete in the range of the UCDs, so we might expect one more to be found in this field. We recently observed the remaining objects, so we will be close to $100 \%$ complete.

M. Fall: For "normal' elliptical galaxies, there are fairly tight correlations between their central colors (or metallicities) and velocity dispersions and their total luminosities. If the outer envelope of a normal elliptical were stripped off, leaving behind a compact core, its central properties would change very little while its total luminosity could be drastically reduced. Thus, the surviving compact core would sit well off the normal color-velocity dispersion-luminosity relations and the amount by which it does so should provide an indication of how much mass has been stripped off. It would be very interesting to make such comparisons for your newly found compact objects.

\section{Drinkwater: Thank you for your suggestion - I shall give it a go.}

E. Telles: In samples of HII galaxies, less than $10 \%$ of the objects are extreme cases of very compact, unresolved galaxies. Have you given any thought to the possibility that these UCDs are faded, evolved versions of these extreme HII galaxies?

M. Drinkwater: This is an interesting suggestion but I suspect the HII galaxies are much larger systems than the UCDs. If fading is important then they are more likely to correspond to the ultraluminous star clusters found in some recent mergers of galaxies. I should note that our FCSS survey has also detected unresolved HII field galaxies behind the Fornax cluster but these have $L_{*}$ lumi- 
nosities.

H. Zinnecker: I find it quite plausible that your objects are nuclei of dE, N galaxies stripped of their low surface brightness envelopes. If this is true, one would expect several such objects around M87, which is known to be surrounded by hundreds of $\mathrm{dE}, \mathrm{N}$ galaxies. Has anyone looked at the Virgo cluster center to find similar objects?

$M$. Drinkwater: We haven't looked in Virgo yet, but it would clearly be the next place to look, as you suggest. It is possible but difficult to observe Virgo with $2 \mathrm{dF}$ from the AAT. It would probably be necessary to do some color-magnitude pre-selection to make the observations more efficient. 